\title{
Offspring toxicity of silver nanoparticles to Arabidopsis thaliana flowering and floral development
}

\author{
Mingjing Ke ${ }^{\mathrm{a}}$, Yan $\mathrm{Li}^{\mathrm{a}}$, Qian $\mathrm{Qu}^{\mathrm{a}}$, Yizhi Ye ${ }^{\mathrm{a}}$, W.J.G.M. Peijnenburg ${ }^{\mathrm{b}, \mathrm{c}}$, Zhenyan Zhang ${ }^{\mathrm{a}}$, \\ Nuohan $\mathrm{Xu}^{\mathrm{a}}$, Tao $\mathrm{Lu}^{\mathrm{a}}$, Liwei Sun ${ }^{\mathrm{a}}$, Haifeng Qian ${ }^{\mathrm{a}, \mathrm{d}, *}$ \\ ${ }^{a}$ College of Environment, Zhejiang University of Technology, Hangzhou 310032, PR China \\ ${ }^{\mathrm{b}}$ Institute of Environmental Sciences (CML), Leiden University, RA Leiden 2300, the Netherlands \\ ${ }^{\mathrm{c}}$ National Institute of Public Health and the Environment (RIVM), Center for Safety of Substances and Products, P.O. Box 1, Bilthoven, the Netherlands \\ ${ }^{\mathrm{d}}$ State Key Laboratory of Desert and Oasis Ecology, Xinjiang Institute of Ecology and Geography, Chinese Academy of Sciences, Urumqi, 830011, PR China
}

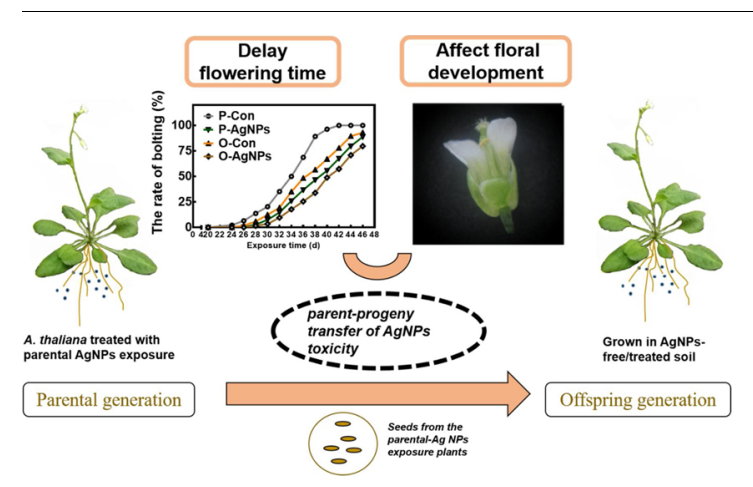

\section{A R T I C L E I N F O}

Editor: R. Debora

Keywords:

Arabidopsis thaliana

Silver nanoparticles

Floral organ

Flowering time

Offspring toxicity

\begin{abstract}
A B S T R A C T
Many studies have considered silver nanoparticles (AgNPs) cytotoxicity to mammalian and human cell lines and plant growth. However, only few studies considered toxic effects of AgNPs on plant offspring, especially on flowering. Arabidopsis thaliana was treated with $12.5 \mathrm{mg} / \mathrm{kg}$ AgNPs employing parental-(P-AgNPs) and offspringgeneration (O-AgNPs) exposure to study the effects of AgNPs on flowering and floral development. Exposure to P-AgNPs was found to significantly decrease petal and pollen viability and subsequently reduced pod production. The inhibition of $A$. thaliana vegetative growth caused by P-AgNPs exposure was transferred to offspring and even became more severe in the O-AgNPs group. Further, the transcription of genes related to flowering and floral organ development in P-AgNPs and O-Con plants was downregulated by approximately 10-40\% compared to the transcription in P-Con plants and showed a stronger decrease in the O-AgNPs group to $30-50 \%$ of that in the P-AgNPs group. This resulted in a delay in flowering of 4, 3 and 8 days in P-AgNPs, O-Con and O-AgNPs plants, respectively. Our research shows that the negative effects on floral development can be transferred to the offspring in A. thaliana, which may have significant implications with regard to the risks posed by NPs to food safety and security.
\end{abstract}

\footnotetext{
*Corresponding author at: College of Environment, Zhejiang University of Technology, Hangzhou 310032, PR China.

E-mail address: hfqian@zjut.edu.cn (H. Qian).
} 


\section{Introduction}

Nanoparticles (NPs) are three-dimensional particles with a diameter of less than $100 \mathrm{~nm}$ in at least one dimension. NPs have a wide range of commercial applications, amongst others due to their antimicrobial properties (Dai et al., 2018; Lebedová et al., 2018; Zhang et al., 2018). Silver nanoparticles (AgNPs), the nanomaterials with the highest degree of commercialization, are estimated to be applied in more than 250 products including textiles, cosmetics, deodorants, bandages, contraceptives, sports items, food products and packaging, cleaning solutions and sprays and have additional applications in the life sciences and biotechnology worldwide (Hackenberg et al., 2011; Jiang et al., 2012). Approximately 500 tons of AgNPs are produced each year worldwide (Calderon-Jimenez et al., 2017), part of which is directly disseminated into the environment during all stages of their life cycle, including production, product use and disposal. These emissions raise ecotoxicological concerns (Gottschalk and Nowack, 2011), amongst others because these AgNPs exert cytotoxic and genotoxic effects on a wide range of organisms including microorganisms, algae, fungi, plants, invertebrates, vertebrates, and human cell lines (Chen et al., 2016; Levard et al., 2012; Li et al., 2018; Liu et al., 2017; Qian et al., 2016; Zhu et al., 2018; Lu et al., 2019).

Notably, AgNPs accumulated in sewage sludge or were deposited into soil, with residual concentrations of $\mathrm{Ag}$ ranging from 1.94 to $856 \mathrm{mg} \mathrm{kg}^{-1}$, leading to a range of effects including inhibition or stimulation of plant growth (Chae et al., 2009; Donner et al., 2015). AgNPs in soil can accumulate in plant roots and translocate to the shoots, leaves or fruits of edible plants (Ke et al., 2018; Qian et al., 2013; Yang et al., 2018). Exposure to AgNPs at varying concentrations $(0-5 \mathrm{mg} / \mathrm{kg}$ sand) can cause a dose-dependent decrease in root elongation and a reduction in shoot biomass in Triticum aestivum (Dimkpa et al., 2013). Rui et al. (2017) reported that exposure to different doses of AgNPs (50-2000 mg/kg) could affect peanut yield and quality based on changes in the fatty acid contents in peanut grains. On the other hand, a few studies reported positive effects of AgNPs on plant growth, e.g. low doses of AgNPs (25-50 $\mathrm{mg} \mathrm{kg}^{-1}$ ) have a beneficial impact on leaf area index, leaf number, chlorophyll content, nitrate reductase (NR) activity, and pod yield of Phaseolus vulgaris (Das et al., 2018). However, the genotoxicity of AgNPs in plants remains unknown, and most relevant studies have been performed with animals or with human cells (AshaRani et al., 2009; Patlolla et al., 2015). Our previous studies reported that exposure to $12.5 \mathrm{mg}$ of AgNPs $/ \mathrm{kg}$ of dry soil during the bolting stage could delay flowering and reduce growth and yield at the maturation stage (Ke et al., 2018). Importantly, based on our metabolomics results, the transfer of elementary Ag released from AgNPs from the soil to plant tissue may induce DNA injury in plant cells. Therefore, we hypothesized that the exposure of Arabidopsis thaliana to AgNPs could potentially alter the offspring physiology and development by injuring the parental plant genetic material.

Flower development is a long and complex process and consists of four stages: flowering transition, floral meristem identity, floral organ identity, and floral organ morphogenesis (Smith and Zhao, 2016). Transforming from the vegetative phase to the reproductive phase is extremely important for all flowering plants (Lu et al., 2018a; Xie et al., 2015b). Floral organ primordia initiate within the floral meristem and later give rise to the formation of the sepal, petal, stamen and carpel, which is controlled by homeotic genes (Bommert et al., 2005). Numerous genes required for flower development have been identified by extensive molecular genetic studies in the model species $A$. thaliana. Several environmental stresses (drought, heavy metals and herbicides) were reported to affect plant flowering development (Kitae et al., 2017; Qian et al., 2014). However, little is known about the impact of AgNPs on plant flowering, although Byczynska et al. (2019) found that tulips treated with $100 \mathrm{mg} \mathrm{L}^{-1}$ AgNPs flowered earlier and their growth was promoted. Therefore, in the present study, we examined the effect of long-term (bolting time and reproduction time) parental- and offspring- generation exposure to $12.5 \mathrm{mg} / \mathrm{kg}$ AgNPs. This concentration of AgNPs was proven to cause parental toxicity on $A$. thaliana in our previous report (Ke et al., 2018). The objective of this study is to compare the phytotoxicity between two generations by assessing plant reproduction (pod quality and seed production), flowering time, floral organ structure and transcription of key genes involved in flowering and pollen development in the model dicotyledon plant $A$. thaliana. The findings of this work will help gain insight into the parent-progeny transfer of NP effects in plants and will provide critical data that can be used to inform risk assessment efforts.

\section{Materials and methods}

\subsection{AgNPs preparation}

AgNPs (citrate-coated) were purchased from Shanghai Huzheng Nano Technology Co. LTD (AGS-WMB1000C, Shanghai, China) and suspended in Milli-Q water by sonication $(20 \mathrm{kHz}$ and $100 \mathrm{~W}$ bath at $25^{\circ} \mathrm{C}$ ) for $30 \mathrm{~min}$ using an ultrasonic cleaner. The actual size distribution was determined by means of transmission electron microscopy (TEM, JEM-1200EX, Tokyo, Japan). The TEM micrographs and the resulting size distribution showed a highly uniform dispersion and a size distribution in between 10 and $12 \mathrm{~nm}$ (Fig. S1), which is close to the reported value of $10 \mathrm{~nm}$ at purchase.

\subsection{Plant culture and seeds collection}

A. thaliana seeds (ecotype: Columbia (Col-0)) were sterilized with $75 \%$ ethanol for $1 \mathrm{~min}$ and $2.5 \%$ sodium hypochlorite (V/V) for $15 \mathrm{~min}$ and then rinsed 6 times with sterilized water. The sterilized seeds were vernalized at $4{ }^{\circ} \mathrm{C}$ for 2 days as reported previously (Sun et al., 2016) and subsequently germinated in plates containing Murashige and Skoog (MS) agar medium (containing $30 \mathrm{~g} / \mathrm{L}$ sucrose and $0.8 \%$ agar) without AgNPs. The chamber temperature was maintained at $25 \pm 0.5^{\circ} \mathrm{C}$, and the seedlings were grown under a $12 \mathrm{~h}$ light $/ 12 \mathrm{~h}$ dark cycle at a light intensity of $300 \mu \mathrm{mol} / \mathrm{m}^{2}$ under cool-white fluorescent lights. After two weeks, the aseptic seedlings were transferred from the MS agar medium to one pot containing potting soil consisting of peat soil and vermiculite at a ratio of 3:1 (Sunshine garden, Canada) containing: N-1.92 g/kg, P$0.155 \mathrm{~g} / \mathrm{kg}, \mathrm{K}-22.5 \mathrm{~g} / \mathrm{kg}$. The soil electrical conductivity ranged from 500 to $650 \mu \mathrm{s} / \mathrm{cm}$, and the soil $\mathrm{pH}$ was at a constant value of 5.7 during the whole exposure duration (Fig. S2). Each pot initially contained a total of $60 \mathrm{~g}$ of dry soil (prior to watering). Then, $300 \mathrm{~mL}$ of MS nutrient solution with $2.5 \mathrm{mg} / \mathrm{L}$ AgNPs was added to the potting soil, and the final concentration of AgNPs was $12.5 \mathrm{mg} / \mathrm{kg}$ of dry soil at the beginning of plant growth. This concentration is close to environmentally realistic Ag concentrations as a recent study on biosolids produced at various periods (from 1950 to 2009) in Australia, U.K. and U.S.A. showed $\mathrm{Ag}$ concentrations to range from 4.3 to $332 \mathrm{mg} \mathrm{kg}^{-1}$ (Donner et al., 2015). Eight replicates were used for each treatment along with a control treatment (hereafter, the seedlings obtained after parental AgNPs exposure are abbreviated as P-AgNPs, and the parental control group (no AgNPs exposure) is abbreviated as P-Con). After approximately two months of exposure, when the plants reached the reproductive stage, seeds were collected for the experiments described below.

\subsection{Parental and offspring-generation treatment}

To further understand the genotoxicity of AgNPs, the seeds collected from parental plants were prepared for parental- and offspring-generation exposure. Four treatments were set including parental and offspring plant exposure to an AgNPs concentration of $12.5 \mathrm{mg} / \mathrm{kg}$ of dry soil (P-AgNPs and O-AgNPs plants) and exposure of parental and offspring plants to soil without AgNPs (P-Con and O-Con plants), with eight replicates each. After approximately 30 days of parental- and 

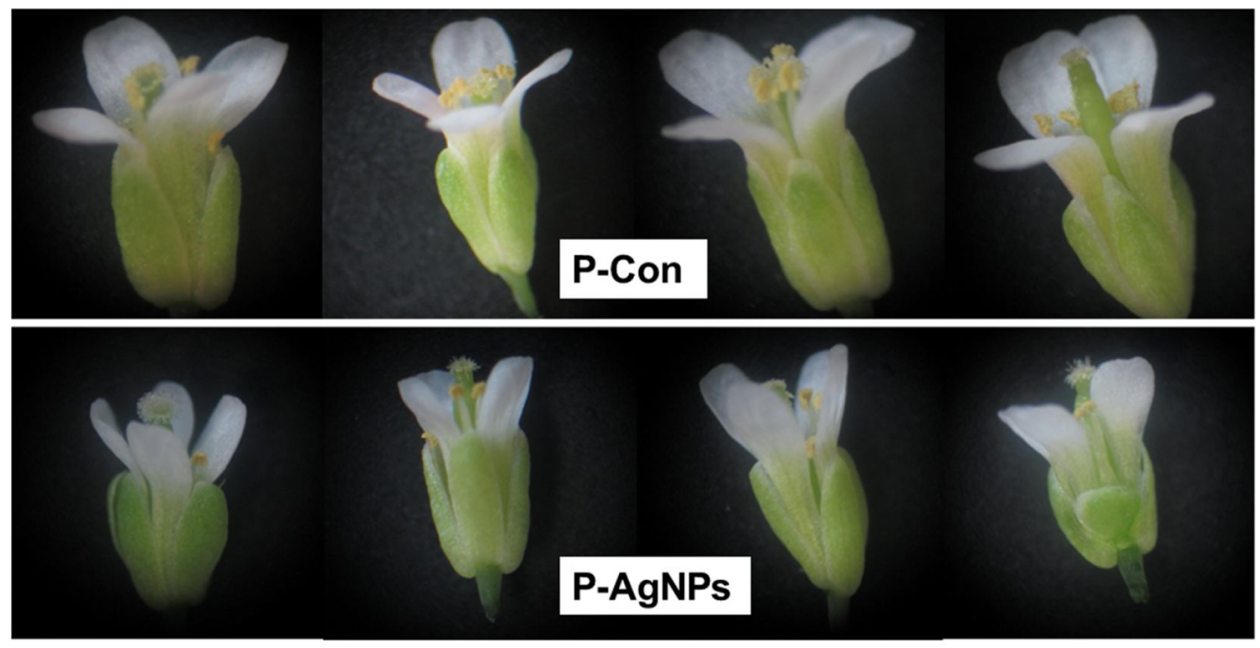

Fig. 1. The effect of parental exposure of $A$. thaliana to $12.5 \mathrm{mg} / \mathrm{kg}$ AgNPs on the floral organ macroscopic structure (P-Con: no AgNPs added in the parental generation, P-AgNPs: $12.5 \mathrm{mg} / \mathrm{kg}$ AgNPs added in the parental generation). Each treatment included four replicates.

offspring-generation exposure, $A$. thaliana seedlings vegetated into the bolting stage and the flowering time was evaluated by using the bolting rate that was calculated when floral buds were visible at the centre of the rosette (Qian et al., 2014). Shoot fresh weight was determined, and the flowering time was recorded.

\subsection{Identification of A. thaliana pods, floral organs and seeds after parental AgNPs exposure at the reproductive stage}

The mature A. thaliana pods were harvested in the P-Con and PAgNPs treatments during the reproductive stage. To identify the quality of pods after parental AgNPs exposure, each separated pod (the largest pods were selected in every treatment) was placed into a tube containing $50 \mathrm{~mL}$ of decolourizing solution (ethanol: acetic acid $=3: 1$ ). The tube was heated in a water bath at $100^{\circ} \mathrm{C}$ until the pod was transparent. The pod was transferred to a clean glass slide with a few drops of autoclaved distilled water and then observed and photographed under a stereoscopic microscope (SZ61-SET, Olympus, Japan). In addition, the intact $A$. thaliana floral organs in the P-Con and PAgNPs treatments were observed under a stereoscopic microscope. The seeds from the P-AgNPs treatment were germinated on MS agar medium in plates as described above. The germination rates were recorded during the first 6 days. After 16 days of cultivation, the shoot fresh weight, seedling taproot length and lateral root number were measured.

\subsection{Effect of parental and offspring soil AgNPs exposure on plant flowering time and floral organs}

During the exposure experiment, plant samples taken after parentaland offspring- generation AgNPs exposure were harvested when the bolting rates reached 50\%. Quantitative real-time PCR (qRT-PCR) was used to measure the transcription of flowering- and floral organ development-related genes. Shoots of $A$. thaliana seedlings were ground up with a ceramic mortar and pestle in liquid nitrogen. Total RNA was isolated from the ground leaves and then reverse transcribed into cDNA using a reverse transcriptase kit (Toyobo, Tokyo, Japan). qRT-PCR was performed with an Eppendorf MasterCycler ep RealPlex thermal cycler (Wesseling-Berzdorf, Germany) using the PCR protocol described in our recent study (Chen et al., 2017; Qian et al., 2011). Note that the sequences of the primer pairs used in the qRT-PCR are listed in Table S1.
2.6. The content of elementary $\mathrm{Ag}$ and the antioxidant enzyme activity (SOD and CAT) in parental and offspring plants

The details of the determination of the silver content and the antioxidant enzyme activity (SOD and CAT) of plant tissue after harvesting are provided in the SI1, SI2.

\subsection{Statistical analysis}

All experiments were conducted with eight replicates except for experiment of quantitative real-time PCR (qRT-PCR), which was replicated four times. We randomly collected one plant out of every replicate for the experiments. The results are shown as mean \pm standard error of the mean (SEM) and the error bars were calculated by using Origin 7.0 (Microcal Software, Northampton, MA). Univariate statistical and correlation analyses were determined by one-way analysis of variance (ANOVA) followed by a post hoc multiple comparison test at the $95.0 \%$ significance level, using the StatView 5.0 software (Lu et al., 2018b).

\section{Results and discussion}

\subsection{The effect of parental AgNPs exposure on A. thaliana floral organ structure and pod quality}

The morphology of the floral organs of $A$. thaliana is presented in Fig. 1. Compared with the morphologies in the P-Con group, both flower size and calyx size decreased, the petals became loose and easily separated from the plant. Besides, the anther size and the amount of pollen decreased after P-AgNPs exposure, which indicated that both stamen and pistil were adversely affected. Therefore, AgNPs exerted an adverse, visually detectable effect on floral organs. In addition, pods in the P-AgNPs group were thinner, shorter and produced small, apparently unfilled seeds compared to those in the P- Con (Fig. 2a). Moreover, both pods weight and length in the P-AgNPs treatment were significantly decreased (by approximately $25 \%$ ) compared to those in the P-Con treatment (Fig. 2b and c), suggesting that they were negatively affected by P-AgNPs exposure.

Floral organ morphogenesis is one of the most important processes in plant reproduction and is vulnerable to various abiotic stresses including heat, cold, and drought (Smith and Zhao, 2016). Lee and Lee (2003) found that pollen tube growth and seed production in A. thaliana were reduced under cold stress. Drought or herbicide stress caused shortened anther filaments, delayed anther development and reduced 


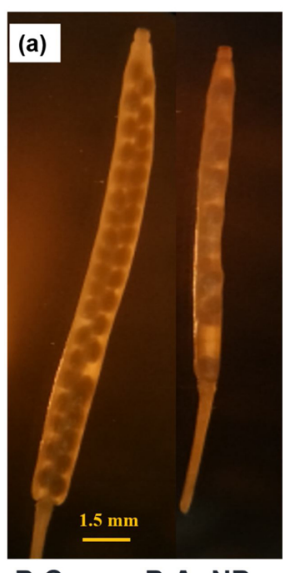

P-Con P-AgNPs
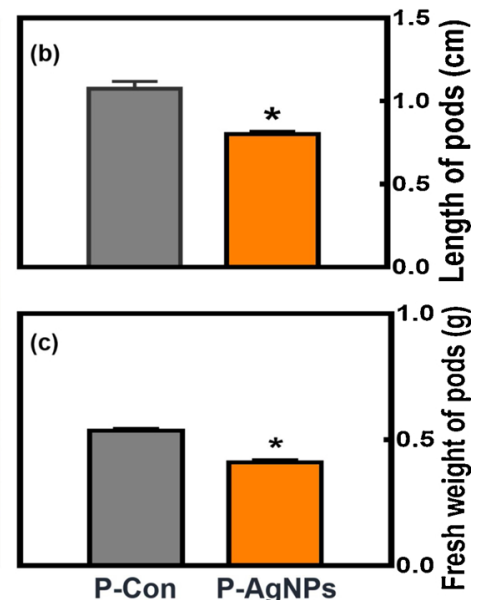

Fig. 2. The effect of parental exposure of A. thaliana to $12.5 \mathrm{mg} / \mathrm{kg}$ AgNPs on pod quality (P-Con: no AgNPs added in the parental generation, P-AgNPs: $12.5 \mathrm{mg} / \mathrm{kg}$ AgNPs added in the parental generation). (a) Pod macroscopic structure. (b) Length of pods. (c) Average fresh weight of 100 pods. * represents statistically significant differences $(p<0.05)$. The values shown are the mean \pm SEM.

pollen viability in $A$. thaliana (Su et al., 2013; Tunc-Ozdemir et al., 2013; Xie et al., 2015b; Qian et al., 2015). However, few studies have investigated the effect of AgNPs on floral organs. Our results showed that floral organs including flower size, calyx size, petals, anther and pollen were adversely affected by AgNPs exposure. Floral organ development is considered as one of the most important processes in plant reproduction, as abnormal floral organs can dramatically decrease the number of pods produced by a plant. Notably, the anther, a part of the stamen, is responsible for the production of pollen and thus plays a crucial role in plant reproduction (Qian et al., 2015). Thus, the abnormal floral organs may dramatically affect pod growth as well as decrease the number of seeds produced by plants, as shown in our study.

\subsection{The effect of parental AgNPs exposure on A. thaliana offspring seed germination and seedling growth}

At the reproductive stage, AgNPs inhibited A. thaliana pod growth and reduced the seed number in the pods. To test the quality of seeds from the P-AgNPs plants, the seed germination rates and offspring growth parameters including shoot fresh weight, taproot length and lateral root number were measured. The germination rate of seeds from P-AgNPs plants was significantly lower than the rate of the seeds collected from unexposed plants (P-Con) after 6 days of cultivation (Fig. 3a). For seedling growth, after 16 days of cultivation, the shoot fresh weight of O-Con plants was significantly reduced to $89 \%$ of the fresh weight of P-Con plants (Fig. 3b). Although the root elongation in O-Con plants was not affected, the lateral root number in these plants decreased by approximately $22 \%$ and $34 \%$ compared to the P-Con plants after 10 days and 16 days of culture, respectively (Fig. $3 \mathrm{c}$ and d). Accordingly, the morphology of $A$. thaliana was also affected, with a decrease in lateral root number and seedling size in the O-Con group compared to the P-Con group (Fig. 3e).

Previous studies have demonstrated that AgNPs can enter plant root cells and inhibit root hair development and root length and that the concentration of $\mathrm{Ag}$ ions released from AgNPs in roots is higher than the Ag concentration in shoots (Ke et al., 2018). In this study, AgNPs significantly inhibited seed germination and lateral root growth in offspring, indicating that the toxic effect of AgNPs can be transferred to offspring. Moreover, Kumari et al. (2009) demonstrated that different concentrations (25, 20, 75 and $100 \mathrm{ppm}$ ) of AgNPs can also penetrate the plant system and may impair stages of cell division, causing extensive damage to DNA and subsequently causing genotoxicity to the root tip of offspring in Allium cepa. This may explain the genotoxicity to roots observed in our experiment.

\subsection{The effect of parental- and offspring-generation AgNPs exposure on A.} thaliana flowering time

To investigate the transgenerational effect of AgNPs on plant growth and flowering in bolting time, shoot fresh weight and flowering time (here, the flowering time is defined as the time needed to reach $50 \%$ bolting) as well as flowering time-related genes in the P-AgNPs and OAgNPs exposure groups were measured. The shoot fresh weight in the P-AgNPs, O-Con and O-AgNPs groups decreased to 55\%, 58\% and $48 \%$ of the shoot fresh weight in the P-Con group (Fig. 4a), respectively, which indicated that the adverse effect of AgNPs on A. thaliana vegetative growth could be transferred to offspring and that O-AgNPs exposure exerted a stronger impact on A. thaliana growth than P-AgNPs exposure. The flowering time in the P-AgNPs group was significantly delayed by approximately 4 days, which is in line with the findings of our previous study (Ke et al., 2018). Notably, flowering time was also delayed by 3 days and 8 days in the O-Con and O-AgNPs groups, respectively (Fig. 4b). The expression of floral integrators that affect terminal flower development, such as AP1, SOC1 and FLY, was significantly decreased to 70-90\% in the P-AgNPs and O-Con groups and to $50-70 \%$ in the O-AgNPs group, respectively, suggesting that offspring AgNPs exposure exerted a much greater impact on flowering time than parental AgNPs exposure. Accordingly, four pathways, namely, the photoperiod pathway, the autonomous pathway, the vernalization pathway and the gibberellin (GA) pathway, were also regulated (Fig. 4c). In the autonomous pathway, both FCA and FY in the OAgNPs group were significantly decreased by $35 \%$ and $41 \%$ compared to the P-Con group, respectively, and AGL24 was significantly increased by $50 \%$. In contrast, only FLY was decreased (by $31 \%$ and $35 \%$ ) in the P-AgNPs and O-Con groups, respectively, suggesting that offspring AgNPs exposure had a stronger effect on the flowering pathways than parental AgNPs exposure. In the P-AgNPs, O-Con and O-AgNPs groups, genes (VRN1, VRN2 and VIN3) in the vernalization pathway were downregulated by $30-50 \%$, and genes (CO, GI and TOC1) in the photoperiod pathway were downregulated to $50-75 \%$ of those in the P-Con group. The tested genes in the GA pathway were not significantly regulated in the P-AgNPs and O-Con groups, while genes (SPY and RGA) in the O-AgNPs group were down- or upregulated by approximately 10-20\% compared to those in the P-Con group. These data showed that the delay in flowering time and changes in four genetic pathways induced by O-AgNPs exposure were significantly greater than those induced by P-AgNPs exposure.

AP1 is a crucial factor to orchestrate a gene network that affects terminal flower development and early flowering phenotypes in Arabidopsis. AP1 is directly activated by FT and LHY (Nilsson et al., 1998; Benlloch et al., 2011). In this study, we demonstrated that parental AgNPs exposure delayed the flowering time by down-regulating AP1 transcription, and this same phenomenon was observed in offspring. It was even strengthened in offspring after AgNPs exposure. Accordingly, the flowering pathways controlled by the integrated flowering genes were affected by parental and offspring with AgNPs exposure. AGL24, an intermediate between SOC1 and LFY, can be upregulated sharply by vernalization to induce floral transition (Michaels et al., 2003). FLC is known to play a central role in regulating the response to vernalization (Ratcliffe et al., 2001). We observed that the transcription of AGL24 was up-regulated in both P- and O-AgNPs exposure, while FLC was up-regulated only in the O-AgNPs group. This indicates that an AgNPs-induced delay of flowering was regulated by the vernalization pathway. Notably, after AgNPs exposure, the genes transcriptional level was not significantly altered in the autonomous and the gibberellin (GA) pathway in the parental generation, but it was affected significantly in the offspring generation. This indicated that 
(a)

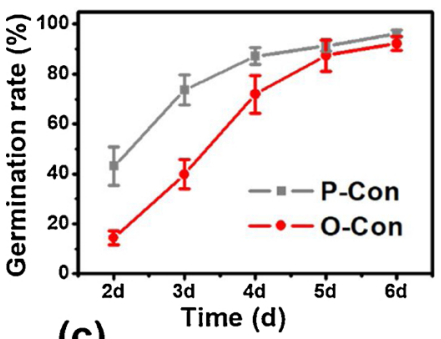

(c)

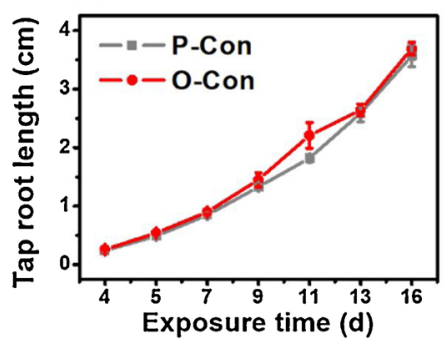

(a)

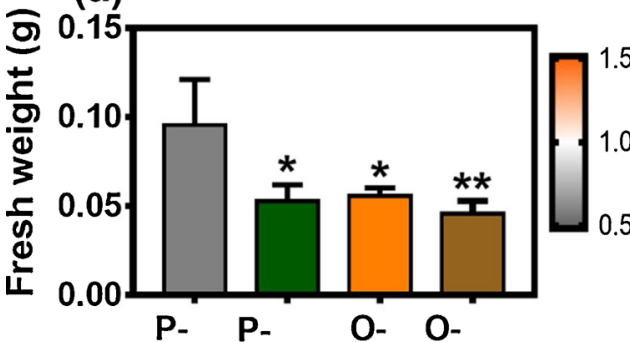

(b) Con AgNPs Con AgNPs

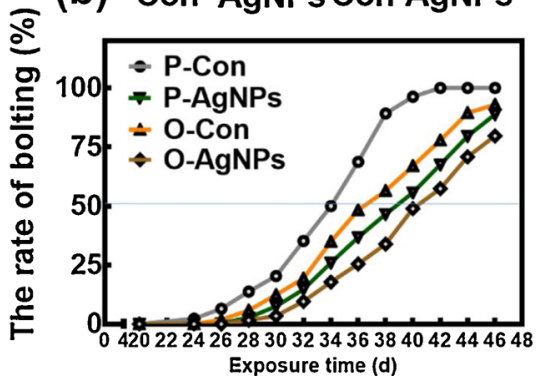

(e)

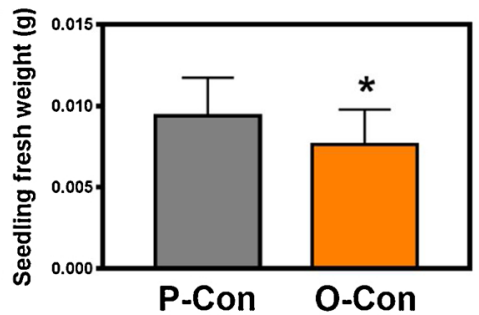

(d)

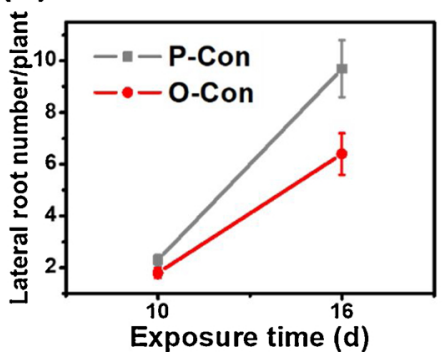

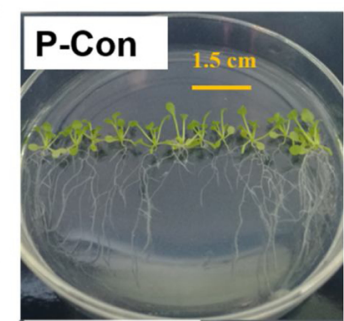

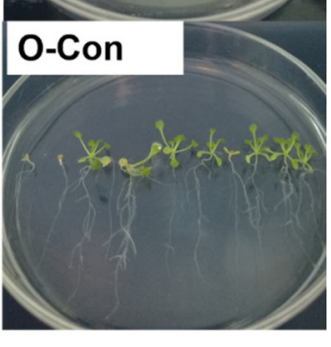

Fig. 3. The effect of parental exposure of $A$. thaliana to $12.5 \mathrm{mg} / \mathrm{kg}$ AgNPs on offspring seed germination and seedling growth (P-Con: no AgNPs added in the parental generation, OCon: seeds from the parental Ag NPs exposure plants were grown in AgNPs-free soil). (a) Offspring seed germination after 6 days of cultivation. (b) Offspring seedling fresh weight after 16 days of cultivation. (c) Offspring taproot length after 16 days of cultivation. (d) Offspring lateral root number after 16 days of cultivation. (e) The morphology of offspring of A. thaliana after 16 days of cultivation. * represents statistically significant differences $(p<0.05)$. The values shown are the mean \pm SEM.

Fig. 4. The effect of parental- and offspringgeneration exposure of $A$. thaliana to $12.5 \mathrm{mg} /$ $\mathrm{kg}$ AgNPs on the fresh weight, flowering time and relative transcription of flowering time-related genes (P-Con: no AgNPs added in the parental generation, P-AgNPs: $12.5 \mathrm{mg} / \mathrm{kg}$ AgNPs added in the parental generation, O-Con: seeds from the parental Ag NPs exposure plants were grown in AgNPs-free soil, O-AgNPs: seeds from the parental Ag NPs exposure plants were grown in AgNPs-treated soil). (a) Fresh weight. (b) Flowering time. (c) Relative transcription of flowering time-related genes in A. thaliana * and ** represent statistically significant differences $(p<0.05)$. The values shown are the mean \pm SEM.
AgNPs can also utilize the autonomous and gibberellin (GA) pathway to induce a delay in flowering when the elemental $\mathrm{Ag}$ concentration reached a certain threshold in the plant seedlings (as more elemental Ag was accumulated in offspring than in parental plants: this result is shown below).

Flowering time, controlled by flowering-related networks, is affected by many environmental stress factors such as drought, herbicides, extreme temperature and pathogen infection as well as intrinsic factors (Itoh and Izawa, 2013; Simpson and Dean, 2002; Qian et al., 2014). Some articles have reported that early or late flowering is a defensive mechanism that is driven by the need to adjust the life histories to encounter suitable environmental conditions (Qian et al., 2014; Ke et al., 2018). Compared with P-AgNPs exposure, O-AgNPs exposure resulted in a greater plant fresh weight inhibition and delay in flowering time in A. thaliana. AgNPs exerted genotoxicity via plant growth inhibition, which may weaken the plant's ability to respond to stress, subsequently causing a greater delay in flowering time in $A$. thaliana offspring than in parents. In general, our results indicated that AgNPs exerted a heritable negative effect on plant growth, late flowering as well as related pathways (floral integrators, the autonomous pathway and the GA pathway), and these negative effects were transferable to offspring and were enhanced by offspring-generation AgNPs exposure.

\subsection{The effect of parental- and offspring-generation AgNPs exposure on A.} thaliana floral organs

The observed morphological changes (as shown in Fig. 1) indicated potential damage caused by P-AgNPs exposure. Thus, we speculated that AgNPs may regulate some floral organ-specification genes. Fig. 5 shows that the transcripts of DYT1, MS1 and MYB103 in the P-AgNPs group were significantly reduced by approximately $19 \%, 10 \%$ and $39 \%$ compared to those in the P-Con group, respectively. MS1 and MYB103 in the O-Con group were downregulated by approximately $20 \%$ compared to those in the P-Con group, while almost all genes, including DYT1, MS1, MS2, AMS and MYB103, in the O-AgNPs group were downregulated to $55-65 \%$ of those in the P-Con group, indicating that AgNPs exerted a transgenerational effect on floral organs and that 


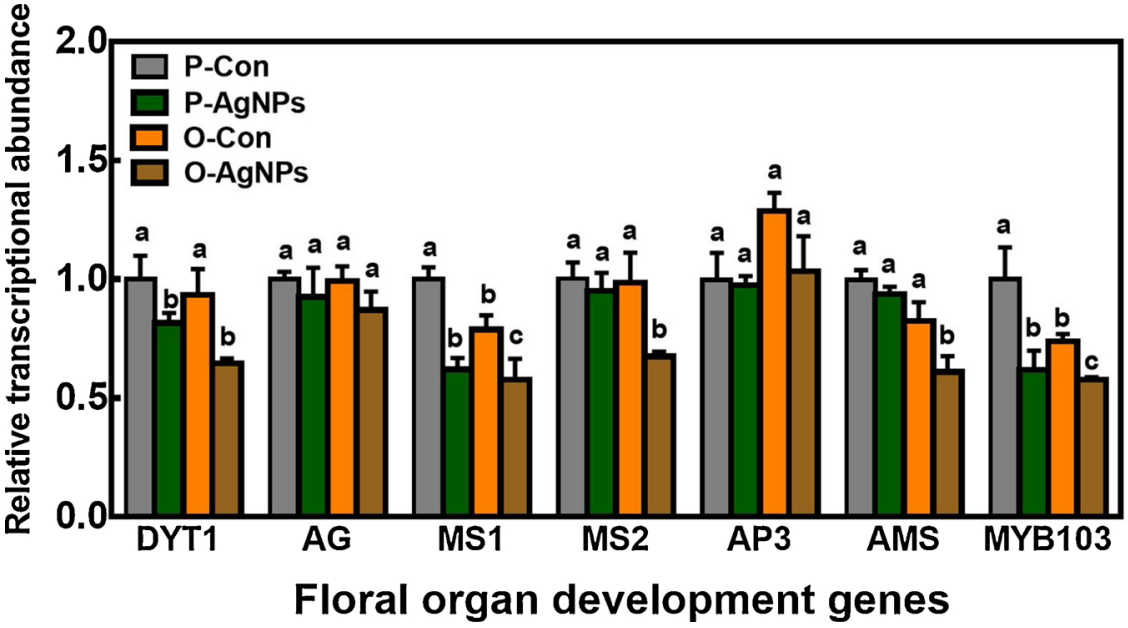

Fig. 5. The effect of parental- and offspring-generation exposure of $A$. thaliana to $12.5 \mathrm{mg} / \mathrm{kg}$ AgNPs on the relative transcription of floral organ development-related genes (P-Con: no AgNPs added in the parental generation, P-AgNPs: $12.5 \mathrm{mg} / \mathrm{kg}$ AgNPs added in the parental generation, O-Con: seeds from the parental Ag NPs exposure plants were grown in AgNPs-free soil, O-AgNPs: seeds from the parental Ag NPs exposure plants were grown in AgNPstreated soil). Different letters represent statistically significant differences. The values shown are the mean $\pm \operatorname{SEM}(n=4)$. offspring exposure elicited a stronger reduction than parental exposure.

DYT1, a crucial component of a genetic network that is involved in A. thaliana tapetum development, lies upstream of at least 22 genes encoding transcription factors and thus regulates the expression of a large number of genes, such as AMS, MYB103, MS1 and MS2 (Zhang et al., 2006; Qian et al., 2014). In our work, the AgNPs-induced downregulation of DYT1 caused a decrease in the transcription of MS1 and MYB103 in the P-AgNPs exposure group, which may have led to the production of anthers that could not easily dehisce to allow pollen dissemination. MS1, AMS and MYB103 are necessary for pollen formation and development, and their suppression inhibits pollen grain production or causes male sterility (Zhu et al., 2008). Therefore, the inhibition of these key genes in A. thaliana exposed to AgNPs may well explain the measured decrease in pollen viability, which finally led to a decrease in the number of seeds per pod. Importantly, these gene alterations also occurred in response to offspring-generation AgNPs exposure. Therefore, AgNPs strongly inhibited floral organ development, primarily via transcriptional regulation, and these negative effects caused by parental AgNPs exposure were transferred to plant offspring and were even strengthened via offspring exposure.

\subsection{The mechanism of AgNPs toxicity to offspring}

To better understand the mechanism of AgNPs toxicity to offspring, we measured the content of elementary Ag and the antioxidant enzyme activity (SOD and CAT) in parental and offspring plants (Fig. 6). Our results showed that AgNPs were not transferred directly from the first generation to the second generation, but the silver content in the offspring AgNPs exposure group was significantly higher than the silver content in the parental AgNPs exposure group, implying that offspring plants were more sensitive to AgNPs. Furthermore, the activity of SOD and CAT also exhibited a higher level in O-AgNPs than in P-AgNPs, indicating a stronger oxidative injury caused by offspring AgNPs exposure (Xie et al., 2015a). Many studies have proven that nanoparticles can enter the root cell of plant and cause physiological and biochemical impacts (negative or positive) on plants. Wang et al. (2016) found that $\gamma-\mathrm{Fe}_{2} \mathrm{O}_{3}$ NPs could enter root cell and was capable of improving iron deficiency and facilitating the growth of watermelon plants. However, Mirzajani et al. (2013) and Mazumdar (2014) demonstrated that AgNPs can penetrate plant cells and cause damage to the cell morphology, vacuoles, cell wall integrity, and likely affect other organelles. Notably, the root tip cells of plants (wheat and A. cepa) treated with AgNPs can exhibit various types of chromosomal aberrations and a decrease in the mitotic index, subsequently interfering with the cells' normal functioning (Kumari et al., 2009; Abdelsalam et al., 2018). This suggests that AgNPs may cause cytotoxicity and genotoxicity to offspring and be an explanation for our findings that AgNPs can affect the quality of pod and floral organs, and even inhibit root development as well as growth of offspring plants. Importantly, the damage to offspring caused by parental AgNPs exposure can lead to an impairment of the plant's ability to respond to stress, and subsequently resulted in a higher accumulation of AgNPs in offspring plant tissue as shown in our work, finally causing greater toxicity on flower development and plant growth of offspring plants. This impact may exhibit a different degree of offspring toxicity due to different sizes of nanoparticles since small particles with larger surface areas may cause higher cellular uptake and toxic responses than larger sized nanoparticles (Wang et al., 2013; Kaveh et al., 2013; Xiang et al., 2019). Therefore, it is of great significant to pay more attention on the impact of different size of AgNPs on offspring plants.
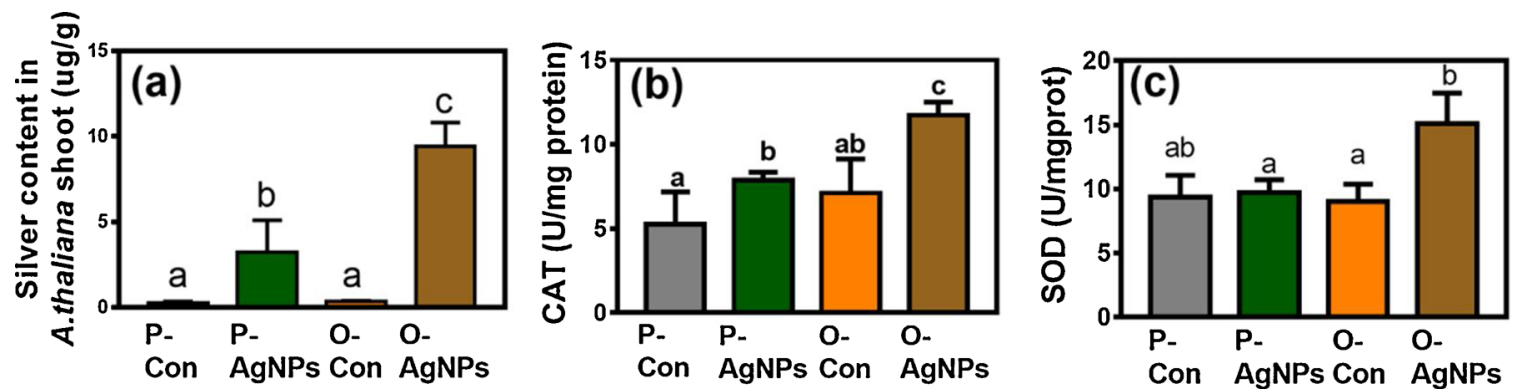

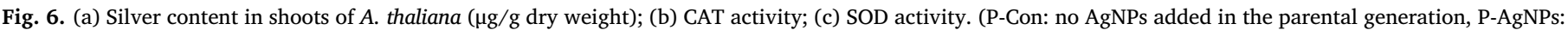

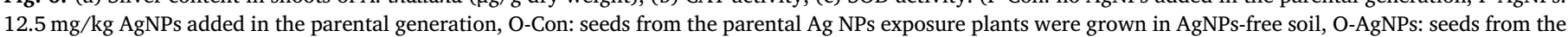

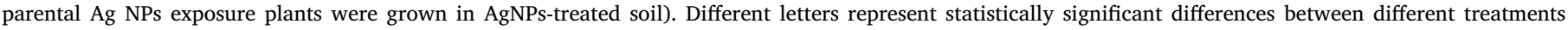
$(\mathrm{p}<0.05)$. The values shown are the mean \pm SEM. 


\section{Conclusion}

Overall, parental AgNPs exposure caused a toxic effect on the floral organs of $A$. thaliana and AgNPs exposure impacted pod quality as well as offspring seed growth. In addition, AgNPs delayed the flowering time by changing the related pathways (the photoperiod pathway, the autonomous pathway and the vernalization pathway) and inhibited pollen formation and pollen development by regulating the transcription of related genes. We demonstrated that all these negative effects on floral development can be transferred to the offspring, although AgNPs themselves were not transferred from the parent to offspring plants. Importantly, exposure of offspring to AgNPs affected plant growth and flower development more strongly than did parental exposure. These findings suggest the need for an integral assessment of the impact of exposure of plants to nanoparticles as plants are a crucial component of environmental and ecological systems.

\section{CRediT authorship contribution statement}

Mingjing Ke: Writing - original draft. Yan Li: Data curation. Qian Qu: Data curation. Yizhi Ye: Methodology. W.J.G.M. Peijnenburg: Writing - review \& editing. Zhenyan Zhang: Visualization. Nuohan Xu: Conceptualization. Tao Lu: . Liwei Sun: Writing - review \& editing. Haifeng Qian: Writing - review \& editing.

\section{Declaration of Competing Interest}

The authors declare that they have no known competing financial interests or personal relationships that could have appeared to influence the work reported in this paper.

\section{Acknowledgments}

This work was financially supported by the Natural Science Foundation of China (21577128, 21777144, 21976161), CAS Pioneer Hundred Talents Program to H.F. Qian, Xingjiang Uighur Autonomous Region Talent Project to H.F. Qian and the European Union of the H2020 funded project PATROLS (Grant code - 760813).

\section{Appendix A. Supplementary data}

Supplementary material related to this article can be found, in the online version, at doi:https://doi.org/10.1016/j.jhazmat.2019.121975.

\section{References}

Abdelsalam, N.R., Abdel-Megeed, A., Ali, H.M., Salem, M.Z.M., Al-Hayali, M.F.A., Elshikh, M.S., 2018. Genotoxicity effects of silver nanoparticles on wheat (Triticum aestivum L.) root tip cells. Ecotox. Environ. Safe 155, 76-85. https://doi.org/10. 1016/j.ecoenv.2018.02.069.

AshaRani, P.V., Mun, G.L.K., Hande, M.P., Valiyaveettil, S., 2009. Cytotoxicity and genotoxicity of silver nanoparticles in human cells. ACS Nano 3, 279-290. https://doi. org/10.1021/nn800596w.

Benlloch, R., Kim, M.C., Sayou, C., Thévenon, E., Parcy, F., Nilsson, O., 2011. Integrating long-day flowering signals: a LEAFY binding site is essential for proper photoperiodic activation of APETALA1. Plant J. 67, 1094-1102. https://doi.org/10.1111/j.1365313X.2011.04660.x.

Bommert, P., Satoh-Nagasawa, N., Jackson, D., Hirano, H.Y., 2005. Genetics and evolution of inflorescence and flower development in grasses. Plant Cell Physiol. 46, 69-78. https://doi.org/10.1093/pcp/pci504.

Byczynska, A., Zawadzinska, A., Salachna, P., 2019. Silver nanoparticles preplant bulb soaking affects tulip production. J. Acta Agric. Scand., Sect. B 69, 250-256. https:// doi.org/10.1080/09064710.2018.1545863.

Calderon-Jimenez, B., Johnson, M.E., Bustos, A.R.M., Murphy, K.E., Winchester, M.R., Baudrit, J.R.V., 2017. Silver nanoparticles: technological advances, societal impacts, and metrological challenges. Front. Chem. 5, 6. https://doi.org/10.3389/fchem. 2017.00006.

Chae, Y.J., Pham, C.H., Lee, J., Bae, E., Yi, J., Gu, M.B., 2009. Evaluation of the toxic impact of silver nanoparticles on Japanese medaka (Oryzias latipes). Aquat. Toxicol. 94, 320-327. https://doi.org/10.1016/j.aquatox.2009.07.019.

Chen, S., Jin, Y.J., Lavoie, M., Lu, H.P., Zhu, K., Fu, Z.W., Qian, H.F., 2016. A new extracellular von Willebrand A domain-containing protein is involved in silver uptake in Microcystis aeruginosa exposed to silver nanoparticles. Appl. Microbiol. Biot. 100, 8955-8963. https://doi.org/10.1007/s00253-016-7728-9.

Chen, S., Li, X.X., Lavoie, M., Jin, Y.J., Xu, J.H., Fu, Z.W., Qian, H.F., 2017. Diclofopmethyl affects microbial rhizosphere community and induces systemic acquired resistance in rice. J. Environ. Sci. China (China) 51, 352-360. https://doi.org/10.1016/ j.jes.2016.06.027.

Dai, Y., Wang, Z., Zhao, J., Xu, L., Xu, L., Yu, X., Wei, Y., Xing, B., 2018. Interaction of $\mathrm{CuO}$ nanoparticles with plant cells: internalization, oxidative stress, Electron transport chain disruption, and toxicogenomic responses. Environ. Sci. Nano 5, 2269-2281. https://doi.org/10.1039/c8en00222c.

Das, P., Barua, S., Sarkar, S., Karak, N., Bhattacharyya, P., Raza, N., et al., 2018. Plant extract-mediated green silver nanoparticles: efficacy as soil conditioner and plant growth promoter. J. Hazard. Mater. 346, 62-72. https://doi.org/10.1016/j.jhazmat. 2017.12.020.

Dimkpa, C.O., McLean, J.E., Martineau, N., Britt, D.W., Haverkamp, R., Anderson, A.J., 2013. Silver nanoparticles disrupt wheat (Triticum aestivum L.) growth in a sand matrix. Environ. Sci. Technol. 47, 1082-1090. https://doi.org/10.1021/es302973y.

Donner, E., Scheckel, K., Sekine, R., Popelka-Filcoff, R., Bennett, J., Brunetti, G., Naidu, R., McGrath, S., Lombi, E., 2015. Non-labile silver species in biosolids remain stable throughout 50 years of weathering and ageing. Environ. Pollut. 205, 78-86. https:// doi.org/10.1016/j.envpol.2015.05.017.

Gottschalk, F., Nowack, B., 2011. The release of engineered nanomaterials to the environment. J. Environ. Monitor. 13, 1145-1155. https://doi.org/10.1039/ c0em00547a.

Hackenberg, S., Scherzed, A., Kessler, M., Hummel, S., Technau, A., Froelich, K., Ginzkey, C., Koehler, C., Hagen, R., Kleinsasser, N., 2011. Silver nanoparticles: evaluation of DNA damage, toxicity and functional impairment in human mesenchymal stem cells. Toxicol. Lett. 201, 27-33. https://doi.org/10.1016/j.toxlet.2010.12.001.

Itoh, H., Izawa, T., 2013. The coincidence of critical day length recognition for florigen gene expression and floral transition under long-day conditions in rice. Mol. Plant 6 , 635-649. https://doi.org/10.1093/mp/sst022.

Jiang, H.S., Li, M., Chang, F.Y., Li, W., Yin, L.Y., 2012. Physiological analysis of silver nanoparticles and $\mathrm{AgNO}_{3}$ toxicity to Spirodela polyrhiza. Environ. Toxicol. Chem. 31, 1880-1886. https://doi.org/10.1002/etc.1899.

Kaveh, R., Li, Y.S., Ranjbar, S., Tehrani, R., Brueck, C.L., Van Aken, B., 2013. Changes in arabidopsis thaliana gene expression in response to silver nanoparticles and silver ions. Environ. Sci. Technol 47, 10637-10644.

Ke, M., Qu, Q., Peijnenburg, W.J.G.M., Li, X., Zhang, M., Zhang, Z., Lu, T., Pan, X., Qian, H., 2018. Phytotoxic effects of silver nanoparticles and silver ions to Arabidopsis thaliana as revealed by analysis of molecular responses and of metabolic pathways. Sci. Total Environ. 644, 1070-1079. https://doi.org/10.1016/j.scitotenv.2018.07. 061.

Kitae, S., Chul, K.H., Seungho, S., Kyung-Hee, K., Jun-Cheol, M., Yoon, K.J., et al., 2017 Transcriptome analysis of flowering time genes under drought stress in maize leaves. Front. Plant Sci. 8, 267. https://doi.org/10.3389/fpls.2017.00267.

Kumari, M., Mukherjee, A., Chandrasekaran, N., 2009. Genotoxicity of silver nanoparticles in Allium cepa. Sci. Total Environ. 407, 5243-5246. https://doi.org/10. 1016/j.scitotenv.2009.06.024.

Lebedová, J., Hedberg, Y.S., Odnevall, I.W., Karlsson, H.L., 2018. Size-dependent genotoxicity of silver, gold and platinum nanoparticles studied using the mini-gel comet assay and micronucleus scoring with flow cytometry. Mutagen. 33, 77-85. https:// doi.org/10.1093/mutage/gex027.

Lee, J.Y., Lee, D.H., 2003. Use of serial analysis of gene expression technology to reveal changes in gene expression in Arabidopsis pollen undergoing cold stress. Plant Physiol. 132, 517-529. https://doi.org/10.1104/pp.103.020511.

Levard, C., Hotze, E.M., Lowry, G.V., Brown, G.E., 2012. Environmental transformations of silver nanoparticles: impact on stability and toxicity. Environ. Sci. Technol. 46, 6900-6914. https://doi.org/10.1021/es2037405.

Li, X.X., Ke, M.J., Zhang, M., Peijnenburg, W.J.G.M., Fan, X.J., Xu, J.H., Zhang, Z.Y., Lu, T., Fu, Z.W., Qian, H.F., 2018. The interactive effects of diclofop-methyl and silver nanoparticles on Arabidopsis thaliana: growth, photosynthesis and antioxidant system. Environ. Pollut. 232, 212-219. https://doi.org/10.1016/j.envpol.2017.09.034.

Liu, G.F., Zhang, M., Jin, Y.J., Fan, X.J., Xu, J.H., Zhu, Y.C., Fu, Z.W., Pan, X.L., Qian, H.F., 2017. The effects of low concentrations of silver nanoparticles on wheat growth, seed quality, and soil microbial communities. Water Air Soil Pollut. Focus. 228, 348. https://doi.org/10.1007/s11270-017-3523-1.

Lu, T., Ke, M.J., Lavoie, M., Jin, Y., Fan, X., Zhang, Z., Fu, Z., Sun, L., Gillings, M., Peñuelas, J., Qian, H., Zhu, Y.G., 2018a. Rhizosphere microorganisms can influence the timing of plant flowering. Microbiome. 6, 231. https://doi.org/10.1186/s40168018-0615-0.

Lu, T., Zhu, Y.C., Xu, J.H., Ke, M.J., Zhang, M., Tan, C.X., Fu, Z.W., Qian, H.F., 2018b. Evaluation of the toxic response induced by azoxystrobin in the non-target green alga Chlorella pyrenoidosa. Environ. Pollut. 234, 379-388. https://doi.org/10.1016/j. envpol.2017.11.081.

Lu, T., Qu, Q., Lavoie, M., Pan, X.J., M.J.G.M, P., Zhou, Z.G., Pan, X.L., Cai, Z.Q., Qian, H.F., 2019. Insights into the transcriptional responses of a microbial community to silver nanoparticles in a freshwater microcosm. Environ. Pollut. https://doi.org/10. 1016/j.envpol.2019.113727.

Mazumdar, H., 2014. Comparative assessment of the adverse effect of silver nanoparticles to Vigna radiata and Brassica campestris crop plants. Int. J. Eng. Res. Appl. 4, 118-124.

Michaels, S.D., He, Y., Scortecci, K.C., Amasino, R.M., 2003. Attenuation of FLOWERING LOCUS C activity as a mechanism for the evolution of summer-annual flowering behavior in Arabidopsis. Proc. Natl. Acad. Sci. U.S.A. 100, 10102-10107. https://doi. org/10.1073/pnas.1531467100.

Mirzajani, F., Askari, H., Hamzelou, S., Farzaneh, M., Ghassempour, A., 2013. Effect of 
silver nanoparticles on Oryza sativa L. And its rhizosphere bacteria. Ecotoxicol. Environ. Saf. 88, 48-54. https://doi.org/10.1016/j.ecoenv.2012.10.018.

Nilsson, O., Lee, I., Blazquez, M.A., Weigel, D., 1998. Flowering-time genes modulate the response to LEAFY activity. Genetics 150, 403-410. https://doi.org/10.1046/j.13652443.1998.00217.x.

Patlolla, A.K., Hackett, D., Tchounwou, P.B., 2015. Genotoxicity study of silver nanoparticles in bone marrow cells of Sprague-Dawley rats. Food Chem. Toxicol. 85, 52-60. https://doi.org/10.1016/j.fct.2015.05.005.

Qian, H.F., Han, X., Peng, X.F., Lu, T., Liu, W.P., Fu, Z.W., 2014. The circadian clock gene regulatory module enantioselectively mediates imazethapyr-induced early flowering in Arabidopsis thaliana. J. Plant Physiol. 171, 92-98. https://doi.org/10.1016/j.jplph. 2013.11.011.

Qian, H.F., Li, Y., Sun, C., Lavoie, M., Xie, J., Bai, X., Fu, Z., 2015. Trace concentrations of imazethapyr (IM) affect floral organs development and reproduction in Arabidopsis thaliana: IM-induced inhibition of key genes regulating anther and pollen biosynthesis. Ecotoxicology 24, 163-171. https://doi.org/10.1007/s10646-014-1369-5.

Qian, H.F., Peng, X.F., Han, X., Ren, J., Sun, L.W., Fu, Z.W., 2013. Comparison of the toxicity of silver nanoparticles and silver ions on the growth of terrestrial plant model Arabidopsis thaliana. J. Environ. Sci. China (China) 25, 1947-1955 CNKI:SUN:HJKB.0.2013-09-028.

Qian, H.F., Wang, R., Hu, H., Lu, T., Chen, X., Ye, H., Liu, W., Fu, Z., 2011. Enantioselective phytotoxicity of the herbicide imazethapyr and its effect on rice physiology and gene transcription. Environ. Sci. Technol. 45, 7036-7043. https:// doi.org/10.1021/es200703v.

Qian, H.F., Zhu, K., Lu, H., Lavoie, M., Chen, S., Zhou, Z., Deng, Z., Chen, J., Fu, Z., 2016. Contrasting silver nanoparticle toxicity and detoxification strategies in Microcystis aeruginosa and Chlorella vulgaris: new insights from proteomic and physiological analyses. Sci. Total Environ. 572, 1213-1221. https://doi.org/10.1016/j.scitotenv. 2016.08.039.

Ratcliffe, O.J., Nadzan, G.C., Reuber, T.L., Riechmann, J.L., 2001. Regulation of flowering in Arabidopsis by an FLC homologue. Plant Physiol. 126, 122-132. https://doi.org/ 10.1002/hep.22785.

Rui, M.M., Ma, C.X., Tang, X.L., Yang, J., Jiang, F.P., Pan, Y., Xiang, Z.Q., Hao, Y., Rui, Y.K., Cao, W.D., Xing, B.S., 2017. Phytotoxicity of silver nanoparticles to Peanut (Arachis hypogaea L.): physiological responses and food safety. ACS Sustain. Chem. Eng. 5, 6557-6567. https://doi.org/10.1021/acssuschemeng.7b00736.

Simpson, G.G., Dean, C., 2002. Flowering - Arabidopsis, the rosetta stone of flowering time? Science 296, 285-289. https://doi.org/10.1126/science.296.5566.285.

Smith, A.R., Zhao, D.Z., 2016. Sterility caused by floral organ degeneration and abiotic stresses in Arabidopsis and cereal grains. Front. Plant Sci. 7, 1503. https://doi.org/10. 3389/fpls.2016.01503.

Su, Z., Ma, X., Guo, H., et al., 2013. Flower development under drought stress: morphological and transcriptomic analyses reveal acute responses and long-term acclimation in Arabidopsis. Plant Cell 25, 3785-3807. https://doi.org/10.1105/tpc.113. 115428.

Sun, C., Chen, S., Jin, Y., Song, H., Ruan, S., Fu, Z., Asad, M.A.U., Qian, H., 2016. Effects of the herbicide imazethapyr on photosynthesis in PGR5- and NDH-deficient Arabidopsis thaliana at the biochemical, transcriptomic, and proteomic levels. J.
Agric. Food Chem. 64, 4497-4504. https://doi.org/10.1021/acs.jafc.6b01699.

Tunc-Ozdemir, M., Tang, C., Ishka, M.R., Brown, E., Groves, N.R., Myers, C.T., Rato, C., Poulsen, L.R., McDowell, S., Miller, G., Mittler, R., Harper, J.F., 2013. A cyclic nucleotide-gated channel (CNGC16) in pollen is critical for stress tolerance in pollen reproductive development. Plant Physiol. 161, 1010-1020. https://doi.org/10.1104/ pp.112.206888.

Wang, J., Koo, Y., Alexander, A., Yang, Y., Westerhof, S., Zhang, Q.B., Schnoor, J.L., Colvin, V.L., Braam, J., Alvarez, P.J.J., 2013. Phytostimulation of poplars and Arabidopsis exposed to silver nanoparticles and $\mathrm{Ag}^{+}$at sublethal concentrations. Environ. Sci. Technol. 47, 5442-5449. https://doi.org/10.1021/es4004334.

Wang, Y.Q., Hu, J., Dai, Z.Y., Li, J.L., Huang, J., 2016. In vitro assessment of physiological changes of watermelon (Citrullus lanatus) upon iron oxide nanoparticles exposure. Plant Physiol. Biochem. 108, 353-360. https://doi.org/10.1016/j.plaphy.2016.08. 003.

Xiang, S.Y., Ma, X.Z., Shi, H., Ma, T., Tian, C., Chen, Y., Chen, H.T., Chen, X., Luo, K., Cai, L., Wang, D.B., Xue, Y., Huang, J., Sun, X.C., 2019. Green synthesis of an alginatecoated silver nanoparticle shows high antifungal activity by enhancing its cell membrane penetrating ability. Plant Physiol. Biochem. 2, 4087-4096. https://doi. org/10.1021/acsabm.9b00590.

Xie, J., Bai, X.C., Lavoie, M., Lu, H.P., Fan, X.J., Pan, X.L., Fu, Z.W., Qian, H.F., 2015a. Analysis of the proteome of the marine diatom phaeodactylum tricornutum exposed to aluminum providing insights into aluminum toxicity mechanisms. Environ. Sci. Technol. 49, 11182-11190. https://doi.org/10.1021/acs.est.5b03272.

Xie, W., Huang, J., Liu, Y., Rao, J., Luo, D., He, M., 2015b. Exploring potential new floral organ morphogenesis genes of Arabidopsis thaliana using systems biology approach. Front. Plant Sci. 6, 829. https://doi.org/10.3389/fpls.2015.00829.

Yang, J., Jiang, F.P., Ma, C.X., Rui, Y.K., Rui, M.M., Adeel, M., Cao, W.D., Xing, B.S., 2018. Alteration of crop yield and quality of wheat upon exposure to silver nanoparticles in a life cycle study. J. Agr. Food Chem. 66, 2589-2597. https://doi.org/10. 1021/acs.jafc.7b04904.

Zhang, W., Sun, Y., Timofejeva, L., Chen, C., Grossniklaus, U., Ma, H., 2006. Regulation of Arabidopsis tapetum development and function by dysfunctional tapetum1 (dyt1) encoding a putative bHLH transcription factor. Dev. 133, 3085-3095. https://doi org/10.1242/dev.02463.

Zhang, Z.Y., Ke, M.J., Qu, Q., Peijnenburg, W.J.G.M., Lu, T., Zhang, Q., Ye, Y.Z., Xu, P.F., Du, B.B., Sun, L.W., Qian, H.F., 2018. Impact of copper nanoparticles and ionic copper exposure on wheat (Triticum aestivum L.) root morphology and antioxidant response. Environ. Pollut. 239, 689-697. https://doi.org/10.1016/j.envpol.2018.04. 066.

Zhu, D., Zheng, F., Chen, Q.L., Yang, X.R., Christie, P., Ke, X., Zhu, Y.G., 2018. Exposure of a soil collembolan to $\mathrm{Ag}$ nanoparticles and $\mathrm{AgNO}_{3}$ disturbs its associated microbiota and lowers the incidence of antibiotic resistance genes in the gut. Environ. Sci. Technol.(October 22). https://doi.org/10.1021/acs.est.8b02825.

Zhu, J., Chen, H., Li, H., Gao, J.F., Jiang, H., Wang, C., Guan, Y.F., Yang, Z.N., 2008. Defective in tapetal development and function 1 is essential for anther development and tapetal function for microspore maturation in Arabidopsis. Plant J. 55, 266-277. https://doi.org/10.1111/j.1365-313X.2008.03500.x. 